
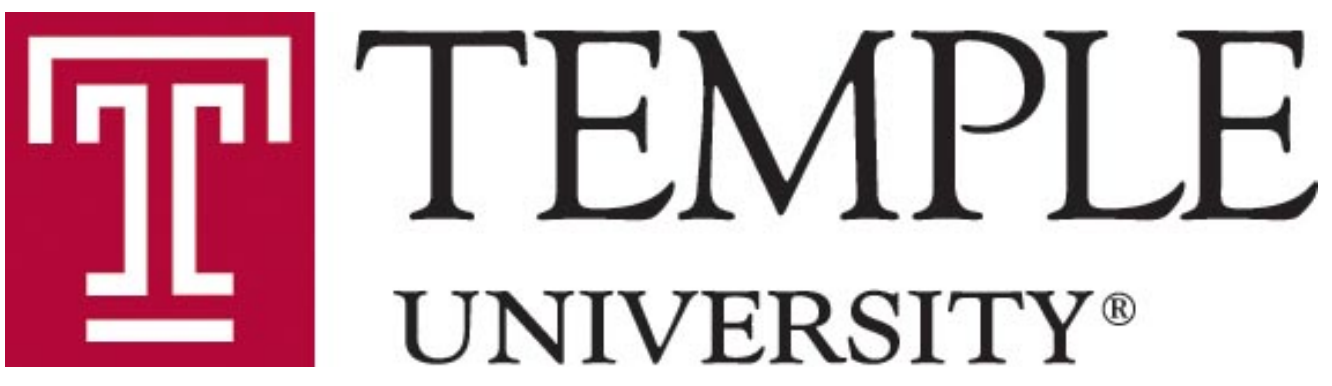

\title{
Local Intergenerational Elasticities
}

\author{
Brantly Callaway \\ Department of Economics \\ Temple University \\ Weige Huang \\ Department of Economics \\ Temple University \\ Department of Economics \\ DETU Working Paper 18-05 \\ August 2018
}

1301 Cecil B. Moore Avenue, Philadelphia, PA 19122

http://www.cla.temple.edu/economics/faculty/detu-working-paper-series/ 


\title{
Local Intergenerational Elasticities*
}

\author{
Brantly Callaway ${ }^{\dagger} \quad$ Weige Huang ${ }^{\ddagger}$
}

August 17, 2018

\begin{abstract}
The intergenerational elasticity (IGE) is the most common parameter reported in the intergenerational mobility literature. This paper proposes a "local" intergenerational mobility parameter (LIGE) that allows the effect of parents' income to vary across different values of parents' income. We also extend this result to an "adjusted" local intergenerational elasticity (ALIGE) which adjusts for differences in the distribution of observed characteristics at different values of parents' income. We develop the asymptotic properties of the LIGE and ALIGE, and apply them to study intergenerational mobility using data from the PSID. We find that the intergenerational elasticity is much larger for low values of parents' income (indicating less mobility) relative to high values of parents' income; adjusting for differences in characteristics reduces the local IGE at all values of parents' income as well as flattening it across different values of parents' income.
\end{abstract}

JEL Codes: J62, C21

Keywords: Intergenerational Income Mobility, Local Linear Estimation, Smooth Coefficient Model

* Code for our method is available as the lige method in the $\mathrm{R}$ package ccfa, and the most recent version can be installed using devtools: : install_github ('WeigeHuangEcon/ccfa')'). This research includes calculations carried out on Temple University's HPC resources and thus was supported in part by the National Science Foundation through major research instrumentation grant number 1625061 and by the US Army Research Laboratory under contract number W911NF-16-2-0189.

${ }^{\dagger}$ Assistant Professor, Department of Economics, Temple University. Email: brantly.callaway@temple.edu

${ }_{\ddagger}^{\ddagger}$ Corresponding Author. $\quad \mathrm{PhD}$ Student, Department of Economics, Temple University. Email: weige.huang@temple.edu 


\section{Introduction}

The intergenerational elasticity (IGE) is the most commonly reported measure of intergenerational income mobility (see, for example, Solon (1992)). It is the coefficient from the regression of the log of child's income on the log of parents' income. Large values of the IGE indicate a relative lack of mobility and small values indicate relatively high mobility. The IGE, however, is a global measure of intergenerational mobility and some researchers have explored how the IGE varies across different values of parents' income (e.g. Landers $\varnothing$ and Heckman (2017)) as a local intergenerational mobility measure. ${ }^{1}$ Researchers studying intergenerational mobility have also been interested in the role of other background characteristics (e.g. race and education) that are correlated with both parents' income and child's income in explaining intergenerational mobility (for example, Bowles and Gintis (2002), Blanden, Gregg, and Macmillan (2007), and Richey and Rosburg (2017)). This paper develops new tools for estimating a local intergenerational elasticity after first adjusting for differences in the distribution of characteristics across different values of parents' income.

The interpretation of local intergenerational income elasticities is somewhat subtle. First, they are local effects and should be interpreted as the effect on average child's income for marginal changes in parents' income. They do not indicate what would happen if parents' income changed dramatically. Also, local intergenerational elasticities are the effect on average child's income (possible after adjusting for differences in covariates) and do not answer questions like how parents' income affects the probability that child's income is below the poverty line. ${ }^{2}$

We propose a semiparametric estimator of the adjusted local intergenerational elasticity that allows for the effects of parents' income and covariates on child's income to change across different values of parents' income. We develop the asymptotic properties of a local linear estimator of the local intergenerational elasticity and our adjusted

\footnotetext{
${ }^{1}$ Also relatedly, Bratsberg et al. (2007) and Björklund, Roine, and Waldenström (2012) group their data by percentiles of parents' income and calculate intergenerational elasticities within groups which is similar to our procedure; Murtazashvili (2012) uses a random coefficients model to allow the effect of parents' income to differ across individuals.

${ }^{2}$ Richey and Rosburg (2016) and Callaway and Huang (2018a) consider how parents' income affects the entire distribution of outcomes though the approach in those papers is substantially different from that of the current paper.
} 
local intergenerational elasticity. Our estimators converge more slowly than parametric estimators though they do not suffer from the curse of dimensionality.

We apply our method to data from the Panel Study of Income Dynamics. Without adjusting for covariates, the local IGE is relatively large and tends to decrease with parents' income. Adjusting for covariates decreases the local IGE across all values of parents' income; however, there is still a strong relationship between child's income and parents' income. Adjusting for covariates also substantially flattens the local IGE across different values of parents' income.

\section{Parameters of Interest}

Let $Y$ denote the log of child's income, $T$ denote the log of parents' income, and $X$ denote a $k \times 1$ vector of covariates. Next, we define our two main objects of interest.

Definition 1. The Local Intergenerational Elasticity (LIGE) is given by

$$
\operatorname{LIGE}(t)=\frac{\partial E[Y \mid T=t]}{\partial t}
$$

$L I G E(t)$ measures the local effect of parents' income on average child's income at a particular value of parents' income $t$. This type of parameter has been considered in Landers $\varnothing$ and Heckman (2017). We are also interested in the effect of parents' income on child's income after adjusting for differences in the distribution of observed characteristics that are related to child's income (e.g. parents with high income are likely to have relatively high education as well) across different values of parents' income. Note that here we are not attempting to establish the causal effect of parents' income; rather, we are trying to imagine what average child's income would be if the return to observed characteristics were held fixed but the distribution of characteristics was changed to be the same as the distribution of characteristics for all individuals in the population.

Next, note that the observed average child's income conditional on parents' income is 
given by

$$
E[Y \mid T=t]=\int_{\mathcal{X}} E[Y \mid T=t, X=x] d F_{X \mid T}(x \mid t)
$$

which holds by the law of iterated expectations and where $\mathcal{X}$ denotes the support of $X$. We consider the counterfactual average outcome conditional on parents' income where the return to characteristics and parents' income is held fixed but the distribution of observed characteristics (conditional on $T=t$ ) is changed to be the distribution of characteristics for the entire population; that is

$$
E^{C}[Y \mid T=t]=\int_{\mathcal{X}} E[Y \mid T=t, X=x] d F_{X}(x)
$$

Given this counterfactual, we define our main parameter of interest next.

Definition 2. The Adjusted Local Intergenerational Elasticity (ALIGE) is given by

$$
A L I G E(t)=\frac{\partial E^{C}[Y \mid T=t]}{\partial t}
$$

$A L I G E(t)$ corresponds to $L I G E(t)$ except that it occurs after adjusting for differences in the distribution of covariates across different values of parents' income. Our next aim is to develop a flexible model for $E[Y \mid X, T]$ in order to ultimately estimate the $A L I G E$. We make the following assumption

Assumption 1 (Smooth Coefficient Model).

$$
Y=X^{\prime} \beta(T)+U
$$

Assumption 1 is key for implementing our method. This type of semiparametric model is called a smooth coefficient model (see, for example, Li, Huang, Li, and Fu (2002) and Cai, Fan, and Li (2000) as well as Callaway and Huang (2018b) for a similar model in the context of decompositions with a continuous treatment). A leading alternative 
idea would be to estimate $E[Y \mid T, X]$ nonparametrically and plug in these estimates to obtain estimates of the ALIGE. With the moderate amount of data typically available in applications this approach is not likely to be feasible as it suffers from the curse of dimensionality. ${ }^{3}$ Even in a case like ours where most of the covariates are discrete, splitting the sample for each possible combination of the discrete variables and then employing nonparametric estimation does not appear to be a feasible strategy either due to sample sizes being extremely small within some cells. It is straightforward to handle this case with our approach though. Another alternative would be to assume that the conditional expectation follows some particular parametric model, but it seems challenging in practice to specify the right functional form; in particular, for the derivative of $E[Y \mid T, X]$ to depend on $X$, the functional form must include interactions between $T$ and $X$ which may be difficult to choose appropriately. Our approach, on the other hand, is quite flexible. We allow the effect of covariates and parents' income to depend on the value of parents' income. For example, the effect of parents' education on child's income can vary across different values of parents' income.

The next result characterizes the ALIGE under Assumption 1.

Proposition 1. Under Assumption 1,

$$
A L I G E(t)=E[X]^{\prime} \frac{\partial \beta(t)}{\partial t}
$$

Proof. First, notice that under Assumption 1,

$$
\begin{aligned}
E^{C}[Y \mid T=t] & =\int_{\mathcal{X}} x^{\prime} \beta(t) d F_{X}(x) \\
& =E[X]^{\prime} \beta(t)
\end{aligned}
$$

Taking the derivative with respect to $t$ implies the result.

Our approach exploits the uniqueness of $T$ among the set of conditioning variables.

\footnotetext{
${ }^{3}$ The curse of dimensionality would be somewhat mitigated from integrating out $X$; however, the fully nonparametric approach is still likely to be difficult to carry out in practice.
} 
Importantly, unlike the fully nonparametric approach, our approach will not suffer from the curse of dimensionality. Our estimator will converge at a slower rate than parametric estimators, but its rate will not slow down due to adding more covariates.

\section{Estimation}

Estimating the LIGE is relatively straightforward. We use local linear kernel regression and an estimate of the derivative is given by the (local) coefficient on the linear term (also notice that the results for the LIGE are a special case of the results for the ALIGE by taking $X$ to only include a constant). For estimating the ALIGE, notice that a first order Taylor approximation of the model in Assumption 1 around $t$ implies

$$
Y \approx X^{\prime} \beta(t)+(T-t) X^{\prime} \frac{\partial \beta(t)}{\partial t}+U
$$

Then, a local linear estimator of $(\beta(t), \partial \beta(t) / \partial t)$ is given by

$$
\left(\begin{array}{c}
\hat{\beta}(t) \\
\frac{\partial \beta(t)}{\partial t}
\end{array}\right)=\left(\mathbf{X}^{\prime} \mathbf{K}(t) \mathbf{X}\right)^{-1} \mathbf{X}^{\prime} \mathbf{K}(t) \mathbf{y}
$$

where $\mathbf{X}$ is an $n \times 2 k$ matrix (where $k$ is the dimension of $X$ ) with the $i$ th row given by $\mathbf{X}_{i}=\left(X_{i}^{\prime},\left(T_{i}-t\right) X_{i}^{\prime}\right)$ and $\mathbf{K}(t)$ is an $n \times n$ diagonal matrix whose $i$ th diagonal element is given by $K_{h}\left(T_{i}-t\right)=K\left(\left(T_{i}-t\right) / h\right)$ where $K$ is a kernel (satisfying some regularity conditions; in practice, we use a trimmed Gaussian kernel though other choices are possible) and $h$ is a bandwidth.

Then, one can estimate the ALIGE as follows

$$
\widehat{A L I G} E(t)=\left(\frac{1}{n} \sum_{i=1}^{n} X_{i}\right)^{\prime} \frac{\partial \hat{\beta(} t)}{\partial t}
$$

In practice, we estimate $L I G E$ and $A L I G E$ over a grid of $L$ possible values for $t$ given by $t^{*}=\left(t_{1}, t_{2}, \ldots, t_{L}\right)$. 


\subsection{Asymptotic Theory}

This section develops the limiting distribution of the LIGE and the ALIGE.

For estimating the LIGE, first let $Y=g(T)+\epsilon$ where $g(t)=E[Y \mid T=t]$. Under standard regularity conditions for local linear estimators (see, for example, Li and Racine (2007)[Theorem 2.7]), ${ }^{4}$ one can show that

$$
\begin{aligned}
n^{1 / 2} h^{3 / 2}(\widehat{L G} E(t)-L I G E(t)) & =\frac{1}{\kappa_{2} f_{T}(t)} n^{-1 / 2} h^{-3 / 2} \sum_{i=1}^{n}\left(T_{i}-t\right) K_{h}\left(T_{i}-t\right) \epsilon_{i} \\
& \stackrel{d}{\rightarrow} N\left(0, V_{L}\right)
\end{aligned}
$$

with $V_{L}=f_{T}^{-1}(t) \kappa_{2}^{-2} \kappa_{22} E\left(\epsilon^{2} \mid t\right)$ and where $f_{T}(t)$ is the marginal density of $T, \kappa_{2}=$ $\int v^{2} K(v) d v$, and $\kappa_{22}=\int v^{2} K^{2}(v) d v$.

Next, for the ALIGE, consider

$$
A \widetilde{L I G} E(t)=E[X]^{\prime} \frac{\widehat{\partial \beta(t)}}{\partial t}
$$

$\widehat{A L I G} E$ and $\widetilde{A L I G} E$ are asymptotically equivalent because $n^{-1} \sum_{i=1}^{n} X_{i}$ converges to $E[X]$ faster than the terms that we estimate nonparametrically. Thus, the asymptotic behavior of $A \widehat{L I G} E$ is driven by the behavior of the local linear term. Under standard regularity conditions for smooth coefficient models (see Cai, Fan, and Yao (2000) and Li, Huang, Li, and Fu (2002)) one can therefore show that

$$
\begin{aligned}
n^{1 / 2} h^{3 / 2} & (A \widehat{L I G E}(t)-A L I G E(t)) \\
& =n^{-1 / 2} h^{-3 / 2} \sum_{i=1}^{n} E[X]^{\prime}\left[\kappa_{2} f_{T}(t) E\left[X X^{\prime} \mid t\right]\right]^{-1} X_{i}\left(T_{i}-t\right) K_{h}\left(T_{i}-t\right) U_{i} \\
& \stackrel{d}{\rightarrow} N\left(0, V_{A}\right)
\end{aligned}
$$

where $V_{A}=f_{T}^{-1}(t) \kappa_{2}^{-2} \kappa_{22} E[X]^{\prime} E\left[X X^{\prime} \mid t\right]^{-1} E\left[X X^{\prime} U^{2} \mid t\right] E\left[X X^{\prime} \mid t\right]^{-1} E[X]$. In practice, we

\footnotetext{
${ }^{4}$ Practically, the most important regularity condition is on the bandwidth. We use cross-validation to choose the bandwidth. In practice, this will "undersmooth" for the derivative term in the local linear estimator leading to the bias term going to zero asymptotically.
} 
Figure 1: The LIGE and ALIGE as a Function of Parents' Income

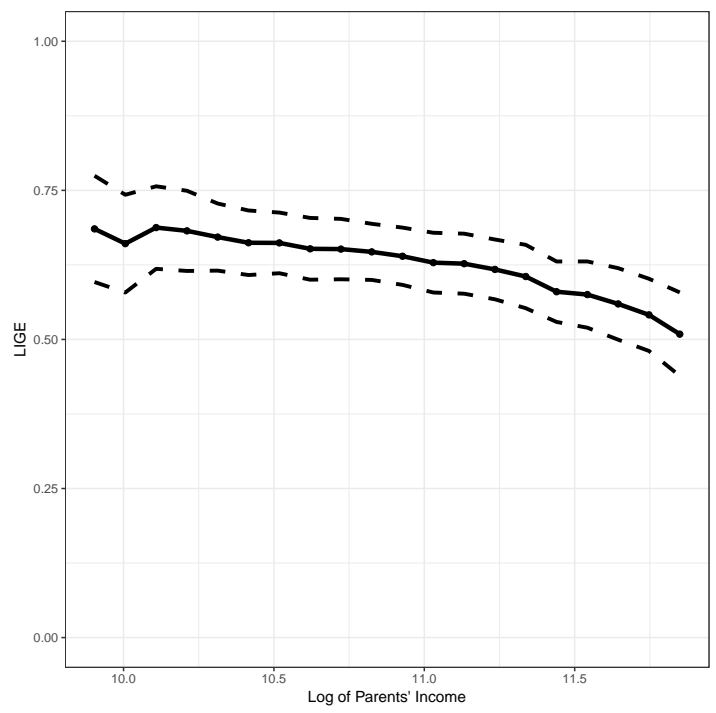

LIGE

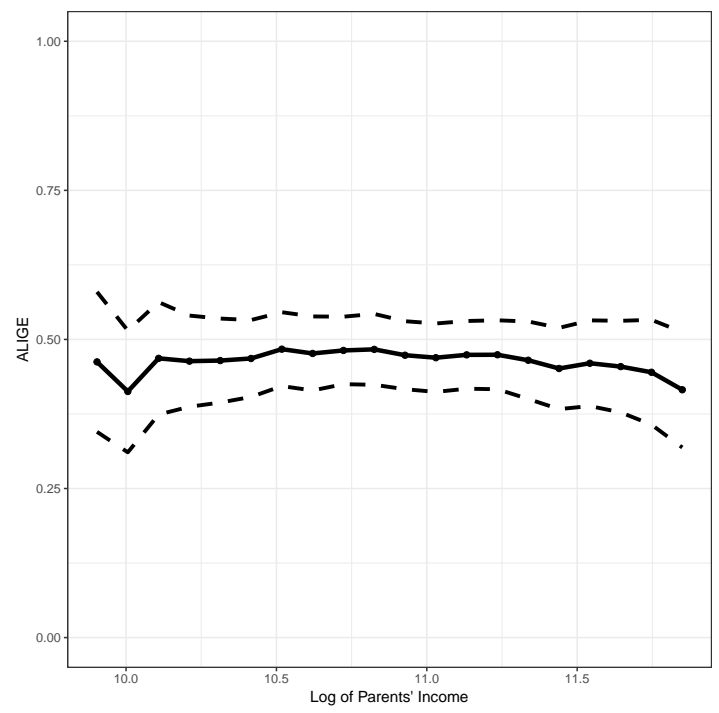

ALIGE

Notes: The left panel plots the LIGE and the right panel plots the ALIGE. In each panel, the dashed lines are pointwise 95\% confidence intervals computed using the wild bootstrap with 500 iterations.

Sources: Panel Study of Income Dynamics, as described in text

carry out pointwise inference using the wild bootstrap.

\section{Application}

We use data from Callaway and Huang (2018a) which comes from the Panel Study of Income Dynamics (PSID). The data consists of 3,630 child-parent measures of permanent income along other characteristics including child's gender and birth year, and the family head's gender, race, educational attainment, and veteran status. See Callaway and Huang (2018a) for a detailed discussion of this data as well as summary statistics for the dataset. Regressing the log of child's income on the log of parents' income results in an estimated IGE of 0.603 .

Our main results are presented in Figure 1. We estimate the LIGE and ALIGE over a grid of twenty equally spaced values of $t$ ranging from $\log (20,000)$ (roughly equal to the poverty line) to $\log (140,000)$. Without adjusting for differences in covariates, the LIGE is equal to 0.69 for children whose parents' income was $\$ 20,000$. It declines substantially in parents' income. For children whose parents income was $\$ 140,000$, the LIGE is 0.51 . 
These results suggest that the effect of parents' income on child's income varies across different values of parents' income; standard measures of intergenerational mobility such as the IGE cannot show this type of heterogeneity.

Adjusting for covariates somewhat diminishes estimates of local intergenerational elasticities. However, even after adjusted for differences in observed covariates across different values of parents' income, the link between child's and parents' income is still strong. For children whose parents' income was $\$ 20,000$, the ALIGE is estimated to be 0.46 . Interestingly, adjusting for covariates also substantially flattens estimated local intergenerational elasticities. The point estimates of the ALIGE are very similar across all values of parents' income (perhaps somewhat declining) and we cannot reject that the ALIGE is constant for all values of parents' income.

\section{Conclusion}

This paper has developed new "local" measures of intergenerational elasticities that allow for the researcher to adjust for differences in the distribution of characteristics across different values of parents' income. We developed a flexible semiparametric estimator of the adjusted local IGE and studied its properties. We found that our adjusting for covariates decreased the local IGE at all values of parents' income and tended to flatten the local IGE as well.

There are many interesting possible extensions of the current framework. First, it would be interesting to develop formal tests that the LIGE or ALIGE are constant across all values of parents' income which could be accomplished using results from the specification testing literature (for example, Hardle and Mammen (1993) and Zheng (1996), among many others); or, relatedly, to develop uniform confidence bands for the LIGE or ALIGE. Second, one could develop tests for whether the LIGE and ALIGE are equal at particular values of parents' income or across all values of parents' income. Finally,

it would be relatively straightforward using our approach to examine the role of each covariate in explaining the difference between the LIGE and ALIGE. This would allow 
one to decompose the gap between the LIGE and ALIGE into parts due to, for example, differences between the distribution of race and education at particular values of parents' income and the overall distribution of race and education. We leave these extensions to future work.

\section{References}

[1] Björklund, Anders, Jesper Roine, and Daniel Waldenström. "Intergenerational top income mobility in Sweden: Capitalist dynasties in the land of equal opportunity?" Journal of Public Economics 96.5 (2012), pp. 474-484.

[2] Blanden, Jo, Paul Gregg, and Lindsey Macmillan. "Accounting for intergenerational income persistence: Noncognitive skills, ability and education". The Economic Journal 117.519 (2007), pp. C43-C60.

[3] Bowles, Samuel and Herbert Gintis. "The inheritance of inequality". Journal of economic Perspectives 16.3 (2002), pp. 3-30.

[4] Bratsberg, Bernt, Knut Røed, Oddbjørn Raaum, Robin Naylor, Markus Jäntti, Tor Eriksson, and Eva Österbacka. "Nonlinearities in intergenerational earnings mobility: Consequences for cross-country comparisons". The Economic Journal 117.519 (2007), pp. C72-C92.

[5] Cai, Zongwu, Jianqing Fan, and Runze Li. "Efficient estimation and inferences for varying-coefficient models". Journal of the American Statistical Association 95.451 (2000), pp. 888-902.

[6] Cai, Zongwu, Jianqing Fan, and Qiwei Yao. "Functional-coefficient regression models for nonlinear time series". Journal of the American Statistical Association 95.451 (2000), pp. 941-956.

[7] Callaway, Brantly and Weige Huang. "Distributional effects of a continuous treatment with an application on intergenerational mobility". Working Paper. 2018. 
[8] Callaway, Brantly and Weige Huang. "Semiparametric estimation of Oaxaca-Blinder decompositions with continuous groups". Working Paper. 2018.

[9] Hardle, Wolfgang and Enno Mammen. "Comparing nonparametric versus parametric regression fits". The Annals of Statistics 21.4 (1993), pp. 1926-1947.

[10] Landers $\varnothing$, Rasmus and James J Heckman. "The Scandinavian fantasy: The sources of intergenerational mobility in Denmark and the U.S." The Scandinavian Journal of Economics 119.1 (2017), pp. 178-230.

[11] Li, Qi, Cliff J Huang, Dong Li, and Tsu-Tan Fu. "Semiparametric smooth coefficient models". Journal of Business \& Economic Statistics 20.3 (2002), pp. 412-422.

[12] Li, Qi and Jeffrey Scott Racine. Nonparametric Econometrics: Theory and Practice. Princeton University Press, 2007.

[13] Murtazashvili, Irina. "An alternative measure of intergenerational income mobility based on a random coefficient model". Journal of Applied Econometrics 27.7 (2012), pp. 1161-1173.

[14] Richey, Jeremiah and Alicia Rosburg. "Decomposing joint distributions via reweighting functions: An application to intergenerational economic mobility". Working Paper. 2016.

[15] Richey, Jeremiah and Alicia Rosburg. "Changing roles of ability and education in US intergenerational mobility". Economic Inquiry 55.1 (2017), pp. 187-201.

[16] Solon, Gary. "Intergenerational income mobility in the United States". The American Economic Review (1992), pp. 393-408.

[17] Zheng, John Xu. "A consistent test of functional form via nonparametric estimation techniques". Journal of Econometrics 75.2 (1996), pp. 263-289. 